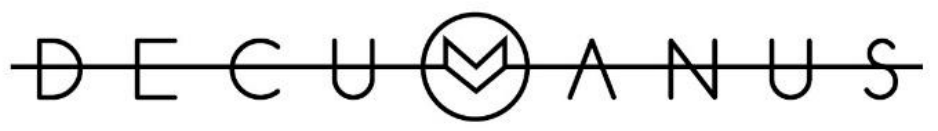

\title{
UNA NUEVA REVOLUCIÓN EN LA MOVILIDAD URBANA: LOS SISTEMAS DE BICICLETAS PÚBLICOS
}

\author{
A NEW REVOLUTION IN URBAN MOBILITY: PUBLIC BICYCLES SYSTEMS
}

\author{
Samuel Rolando González Luna \\ Vinculación \\ Universidad Nacional Autónoma de México, México \\ E-mail: samueltheadler7@hotmail.com \\ Primera versión recibida en: 03 de noviembre, 2015 \\ Última versión recibida en: 15 de julio, 2016
}

\section{Resumen}

La movilidad urbana en la ciudad es una necesidad básica de las personas que debe ser satisfecha, de manera que el esfuerzo que requieran los desplazamientos necesarios para acceder a bienes y servicios no repercuta negativamente en la calidad de vida ni en las posibilidades de desarrollo económico, cultural, educativo, etc. de los ciudadanos. Los viajes o desplazamientos que realiza la población, se han complejizado a causa de la expansión urbana y del crecimiento acelerado de la población que demanda medios y formas de traslados que permitan acceder a los diferentes puntos de un espacio urbano cada vez más extenso, donde los destinos están cada vez más apartados entre sí.

La movilidad es un factor determinante para el desarrollo urbano, territorial y sustentable de las ciudades, el análisis de los desplazamientos urbanos, es preponderante en donde en la actualidad la búsqueda de alternativas enfocadas a hacer a la ciudad sustentable, responsable con el cuidado del medio ambiente, por ello es pertinente realizar un análisis enfocado al diseño e implementación de los sistemas de bicicletas públicas (SBP), el cual constituye un complemento práctico y rápido de la red de transporte público el cual busca mitigar los efectos de la congestión vehicular con beneficios para la salud del usuario.

En el presente artículo se señala la importancia de los SBP, se realiza una revisión de las generaciones de dichos sistemas, así como de los beneficios y desventajas de este tipo de sistemas y finalmente, se debe decir que está surgiendo e impulsando un nuevo paradigma de movilidad urbana no motorizada que está en auge a nivel mundial, el cual se está convirtiendo en una alternativa real de transporte urbano en distancias cortas en la zona central de las ciudades.

Palabras clave: Sistemas de bicicletas públicas, movilidad urbana y territorial, sustentabilidad. 


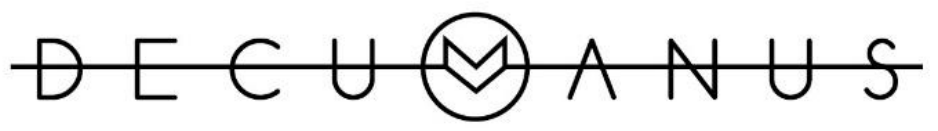

\section{Abstract}

The urban mobility in the city is a basic need for people to be satisfied, and be protected in the effort requiring the necessary movements to access to goods and services does not negatively impact the quality of life or in the development possibilities economic, cultural, educational, etc. citizens trips or journeys that the population, which have become more complex due to urban expansion and rapid growth of the population seeking ways and means of transportation that allow access to different parts of a space Once each larger city where the destinations are increasingly far apart.

Mobility is a key for urban, territorial and sustainable development of cities factor, analysis of urban travel, is predominant today where the search for alternatives aimed to make sustainable, responsible city with the care of the environment is appropriate for them to make a focused on the design and implementation of public bicycle systems (SBP) analysis, which is a practical and easy complement public transport network which seeks to mitigate the effects of traffic congestion with benefits health of the user.

In this article the importance of SBP noted, a review of the generations of such systems is carried out, as well as the benefits and drawbacks of this type of system is finally emerging and promoting a new paradigm of urban motorized mobility are booming worldwide, which are becoming a real alternative urban transportation over short distances in the central cities

Keywords: public bicycle systems, urban and regional mobility, sustainability

\section{Sumario}

- Introducción

- Sistemas de bicicletas públicas

- Características generales e importancia del sistema de bicicletas públicas

- Ventajas de los SBP como medio de movilidad no motorizada (MNM)

- Desventajas de los SBP como medio de movilidad no motorizada (MNM)

- Conclusión: Los SBP, un nuevo paradigma urbano

- Bibliografía

\section{Introducción}

Unikel Luis (1978:119) señala que actualmente para definir a las Zonas Metropolitanas "se han añadido diferentes variables y criterios como: la extensión territorial, la actividad económica, la densidad poblacional, la integración de los municipios hacia la ciudad central y la distancia física entre los centros de la población". Las zonas metropolitanas presentan "problemas en los desplazamientos a 


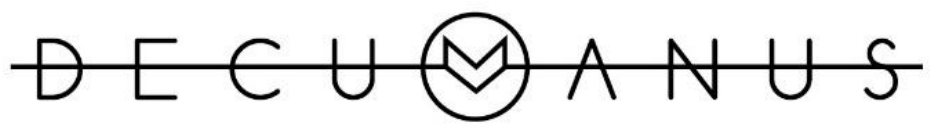

REVISTA INTERDISCIPLINARIA SOBRE ESTUDIOS URBANOS

causa del modelo de crecimiento de la movilidad urbana basado en el uso intensivo de vehículos motorizados privados" (1978:119), es necesario entender que la ciudad central y sus municipios periféricos forman un mismo todo. Por esta razón es apremiante implementar soluciones integrales, sin dejar de lado a los municipios más distantes, los cuales también requieren movilizarse a la ciudad con el objeto de desempeñar sus actividades.

El crecimiento de las áreas urbanas así como la conformación de las zonas metropolitanas "han producido significativos cambios en la movilidad y en los medios de transporte que repercuten en los tiempos y las distancias"(Parrado, Carlos, 2010:22), las cuales, tienen una importante incidencia en la movilidad urbana y en el transporte ya que los habitantes que se localizaban en puntos más apartados de las zonas de trabajo, se ven en la necesidad de "llevar a cabo traslados cada vez más distantes al centro o a la periferia de la ciudad".

Por ello es apremiante la implementación de medios alternativos de transporte urbano, no contaminante y sustentable en las ciudades, Antonio Esteban (1994:39), propone que "un sistema de transporte público sustentable, es aquel que cumple el fin de garantizar una movilidad sostenible", cabe señalar que los gobiernos a nivel global están volteando e impulsando nuevas formas de movilidad.

Actualmente está surgiendo un nuevo paradigma de la movilidad en las ciudades, se fundamenta y valoriza en la representación activa de las personas, así como del conjunto de la sociedad, "en este nuevo enfoque (Moctezuma, 2003:175-189), la movilidad puede ser analizada desde diferentes aspectos, dentro de los cuales destacan: el de los usos y valores, en el cual el movimiento se valora a sí mismo, el de los instrumentos y medios que permite la satisfacción de necesidades y el de los actores".

La movilidad urbana "debe de precisarse dentro del espacio y del territorio" (Hoffmann, Odile, 1997:17), los cuales son dos conceptualizaciones estrechamente relacionadas, y ambos están en continuo cambio y transformación. Hay una relación directa entre las personas y el espacio, por ello el espacio público debe garantizar el libre acceso a toda la población, con el objeto de transportarse y movilizarse, por lo cual existe un vínculo entre el espacio, el territorio y la movilidad. Al definir el concepto de espacio y su incidencia en la movilidad, se puede interpretar desde diferentes perspectivas, para el presente trabajo de manera sucinta se presentan a continuación enfoques que contribuyen a precisar qué es la transformación y la reconfiguración espacial dentro de la movilidad urbana. 


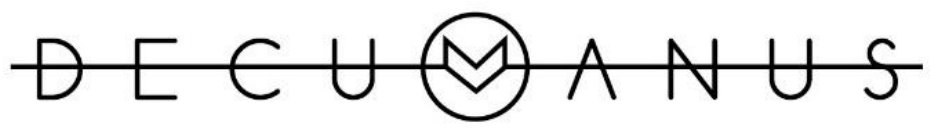

REVISTA INTERDISCIPLINARIA SOBRE ESTUDIOS URBANOS

En primera instancia el trabajo de Massey (2007:79) conceptualiza que "la noción de espacio abarca tres dimensiones; en primer lugar, el espacio es un producto de interrelaciones y de prácticas sociales, en segundo lugar, el espacio es una dimensión que es producto de cosas que están ocurriendo, es producto de interrelaciones, y en tercer lugar, el espacio es una dimensión de multiplicidad, lo cual quiere decir que si no existiera una multiplicidad de cosas no existiría el espacio". Esto es que el espacio es una dimensión de lo social, y al mismo tiempo lo social construye el espacio. La autora concibe el espacio como un proceso, "algo que no está terminado, y es construido todo el tiempo, (Massey 2007:79)". Es importante identificar estas distinciones ya que dentro de los desplazamientos urbanos todos los medios de transporte usan espacio para desplazarse y estacionar durante un determinado periodo de tiempo, cabe resaltar que el coche es el que más espacio consume y de igual forma el más ineficiente en su utilización.

Ahora bien, Odile Hoffmann y Fernando Salmerón (1997:17) señalan que "el espacio no sólo participa como contenedor o soporte material de los procesos sociales, sino también como un elemento activo que influye en la estructuración misma de la sociedad". Para éste trabajo se entiende a la transformación espacial dentro de la movilidad como la serie de cambios que se llevan a cabo dentro de un proceso de transición, encausados a modificar la forma en que un grupo social se apropia del espacio y con esto altera las relaciones endógenas y exógenas. Cuando la estructura de los desplazamientos cambia se reorganiza el espacio y esta reorganización da como resultado una reconfiguración espacial y territorial.

Las transformaciones en la movilidad urbana de las ciudades suponen una serie de cambios de patrones, pueden ser tanto de consumo, de apropiación del espacio, de producción, de establecer relaciones. Ciertamente estas transformaciones ocurren dentro de un espacio y donde los sistemas de transporte consumen una gran cantidad de suelo y espacio, tanto en la circulación como en el estacionamiento en el cual las políticas públicas han privilegiado la movilidad motorizada en gran medida, como lo marcan Gustavo Montañez y Omar Delgado (1997:122) "el espacio es el escenario de las relaciones sociales y de poder".

El posicionamiento de Jordi Borja (2001:81) en torno al espacio, el cual señala que "es necesario concebir al espacio no como una vía para vehículos, sino como un espacio público para la gente, para andar y encontrarse". La continua transformación de la movilidad en el espacio urbano dentro de las ciudades tiene un papel preponderante dentro de su localización, desde el enfoque de las ciencias sociales, no puede concebirse por sí solo, bajo la perspectiva de los estudios regionales 


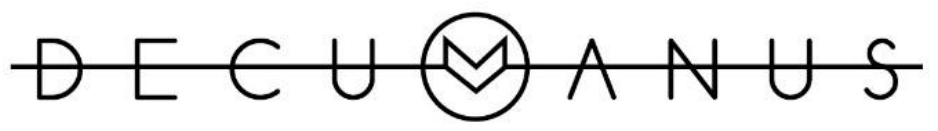

REVISTA INTERDISCIPLINARIA SOBRE ESTUDIOS URBANOS

es una dimensión de la realidad material en donde se llevan a cabo los procesos. Es importante señalar que la disposición de los espacios en las ciudades está determinada por el crecimiento urbano.

El uso de suelo se ha ido especializando con base a su funcionalidad y por la apropiación de las clases dominantes y donde los sistemas viales e infraestructura han sido implementados en gran medida para dichas clases, en éste sentido el espacio público se encuentra determinado por una regulación de la administración pública, la cual ha privilegiado la movilidad motorizada privada, si bien la vía pública es construida para permitir la circulación de personas y vehículos, no para estacionarlos.

En este orden de ideas Borja (2007:30) propone que "el espacio público proviene de la separación legal entre la propiedad privada urbana y la propiedad pública", la noción de espacio público es sinónimo de la calle, lo visible, lo colectivo, la apertura, y el cual se caracteriza por su accesibilidad y una gran gama de actividades, la relación entre los habitantes, la movilidad y el uso del poder se materializan en el espacio urbano el cual ordena la ciudad y le da sentido a la actividad humana.

Para Jordi Borja y Zaida Muxí (2007:30):

El espacio público supone un dominio público, un uso social colectivo y multifuncional, se caracteriza físicamente por su accesibilidad, lo que le hace un factor de centralidad, la calidad del espacio público se podrá evaluar sobre todo por la intensidad y la calidad de las relaciones sociales que facilita, por su fuerza mezcladora de grupos y comportamientos, por su capacidad de estimular de forma simbólica, la expresión y la integración cultural.

Con base en este concepto, podemos afirmar que las grandes vialidades no cumplen con la función de espacio público ya que ocupan una gran cantidad de espacio que está diseñado en función del automóvil lo que limita el uso a los peatones y ciclistas. Con ello se reduce la movilidad de los mismos y se vulnera su derecho a la ciudad.

Otra manera de entender el espacio público es como un determinante de la forma de la ciudad, el cual ordena la estructura del tejido urbano, es un articulador de las áreas urbanas, mediante la creación de espacios de transición con el entorno. Dentro del espacio público, todos deberíamos ser iguales, "éste se ha convertido en un elemento especializado, en un equipamiento más de la ciudad, en una serie de espacios segregados cargados de especificidades, el cual pretende construir tejidos sociales abiertos e igualitarios".

En resumen, es importante definir la relación entre la movilidad y el espacio, ya que continuamente existe una disputa por éste entre peatones, ciclistas y automovilistas dentro del espacio 


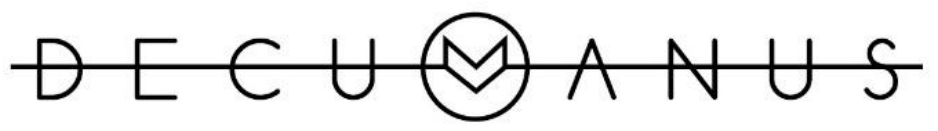

REVISTA INTERDISCIPLINARIA SOBRE ESTUDIOS URBANOS

urbano. Si bien la movilidad contiene conceptos de transporte y convivencia, cuyas relaciones se combinan justamente con el espacio y el lugar en donde se desarrollan. Dentro del tema de estudio en los últimos años la movilidad no motorizada ha cobrado un gran impulso a nivel mundial, la cual ha modificado y reorganizado la estructura del espacio urbano, de las calles y vialidades.

\section{Sistemas de Bicicletas Públicas}

Actualmente se ha hecho indispensable plantear el uso de los Sistemas de Bicicletas Públicos (SBP), como una estrategia de Movilidad no motorizada (MNM), la cual se define como el uso de un medio de transporte el cual no tiene motor y por ende no tiene efectos negativos en el ambiente ni en la salud de las personas. Dentro de los principales medios no motorizados destacan, el uso de la bicicleta, o bien el caminar a pie en trayectos cortos. Cabe enfatizar que los desplazamientos de tipo no motorizado siempre han sido utilizados en la ciudad de México, en donde la introducción de la bicicleta en el siglo XIX, permitió que su uso fuera enfocado a un uso por diversión, convirtiéndose posteriormente en un medio de transporte y de trabajo, de ahí surgió su uso para la distribución de productos de fácil manejo, como lo es el reparto de agua embotellada, pan, comida por mencionar solo algunos.

En 2007 Béllen Duque señalo que la MNM "tiene tanto beneficios a nivel individual como de forma colectiva, su utilización se traduce en un aumento de la calidad de vida y en un cuidado del ambiente" (2007:169), es un medio de transporte accesible y ayuda a disminuir el excesivo uso de vehículos motorizados y modificar las formas de movilidad en la ciudad, proporciona mayor flexibilidad por su peso, su tamaño, etcétera.

Actualmente "los proyectos de carácter público de movilidad, tradicionalmente se han vinculado con los procesos de desarrollo del transporte de tipo motorizado como lo son el metro, metrobús y tren ligero" (Connolly, Priscila, 2010:63), por ello ha generado la exigencia por parte de la población de implementar políticas públicas que den solución eficaz y sustentable, al rubro del servicio del transporte público. Así mismo "se ha intentado transformar el modelo de movilidad en diversas ciudades europeas, latinoamericanas y la ciudad de México no es la excepción" (IDEA, 2012:78). Para ello se deben considerar tres puntos fundamentales: los lugares y espacios viales en donde confluyen tanto la movilidad motorizada y no motorizada, la cultura entendida como un comportamiento distinto de la población en relación al aumento de los problemas de movilidad y las nuevas alternativas, las cuales no deben enfocarse solo en los vehículos motorizados. 


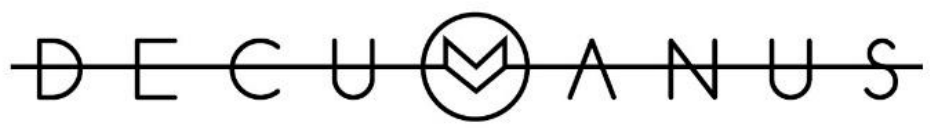

REVISTA INTERDISCIPLINARIA SOBRE ESTUDIOS URBANOS

Es importante definir qué son los sistemas de bicicletas públicas (SBP), ya que éstos son un ejemplo de la MNM, son una variante de las bicicletas tradicionales, en las cuales se les introduce cambios en el diseño y un sistema de préstamo para que sean compartidas por distintos usuarios. "La necesidad de usar la bicicleta surge en diversas ciudades europeas como lo es Holanda con base en la búsqueda de transportes alternativos de carácter sustentable como lo es la MNM, mediante el programa White Bike Plan" (IDEA, 2012:24), como una modalidad de transporte público que recientemente ha comenzado a extenderse en varias ciudades del mundo. En un inicio los sistemas de bicicletas públicas (SBP), "permitían el acceso a una bicicleta de forma gratuita y sin trámites, derivado de ello" (IDEA, 2012:32), el éxito de los programas estaba supeditado a la voluntad de los usuarios para que no fueran a maltratarlas y que no fueran robadas. En efecto, "el uso de bicicletas públicas inició como un proyecto sencillo hasta ir evolucionando a sistemas muy avanzados acorde a las características de cada ciudad" (IDEA, 2012:18).

Fue en los años 60 (NICHES, 2005), cuando se le consideró como una verdadera "alternativa de transporte público el cual trae ventajas en la salud, el ambiente, el espacio y en la economía". Los sistemas de bicicletas públicas urbanas se presentan como programas encaminados a impulsar la MNM en distancias cortas en las ciudades, actualmente son grandes multinacionales de publicidad exterior las que proveen este servicio, "Holanda y Dinamarca son los mejores ejemplos de la puesta en aplicación de este tipo de servicios con un gran éxito entre la población” (IDEA, 2012:22).

El programa europeo NICHES en 2005, para la investigación y desarrollo de políticas sostenibles de transporte, lleva a cabo una clasificación de los SBP catalogándolos en cuatro generaciones de SBP:

1a Generación: se les conoce como sistemas abiertos, surgen en 1968, en Ámsterdam, Holanda, con la instauración del White Bike Plan, por parte de Laurens Hendrikus, tratando de instalar en la capital holandesa 20,000 bicicletas de tipo comunitario. Tuvo como objetivo evidenciar la contaminación ocasionada por los vehículos motorizados, así como enfatizar la mala calidad del transporte público. Se caracterizan por ser sistemas (Barragán, 2010:55) "gratuitos abiertos, sin un control, ni registro, se basó en inundar las calles con bicicletas teñidas de blanco". El fracaso de esta primera generación, fue principalmente debido a la vulnerabilidad, al robo y vandalismo, ya que no existía un espacio específico para devolverlas, si bien el costo era bajo ya que generalmente eran aportaciones de patrocinios o de donaciones.

Ciertamente fue punta de lanza para los SBP, es por ello que posteriormente este tipo de SBP se recomienda implantarlo solo en lugares o áreas controladas de entrada y salida como lo son 


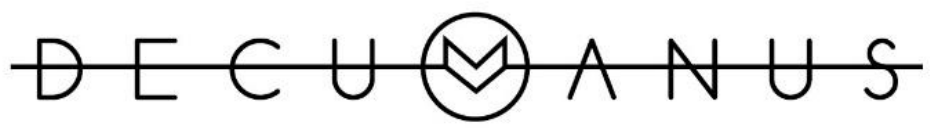

REVISTA INTERDISCIPLINARIA SOBRE ESTUDIOS URBANOS

jardines y universidades, un caso de éxito en la CDMX, es el programa Bicipuma el primero que existió en la Ciudad de México, el cual es de uso exclusivo para la comunidad universitaria dentro del campus de la UNAM, su eficiencia radica en un eficaz control, sensibilización y apropiación.

2 ${ }^{\text {a }}$ Generación; es un sistema con más control, cuenta con lugares específicos para tomar y dejar la bicicleta, si bien como el anterior sistema busca disminuir los niveles de contaminación en el ambiente, de igual forma se piensa como una opción alternativa de movilidad dentro de las grandes ciudades en distancias cortas, aparece en 1995, pero continúa siendo un sistema gratuito, ya que sólo es necesario dejar una fianza muy baja por la utilización de la bicicleta, continúa presentando vulnerabilidad por la falta de seguridad, las únicas medidas de seguridad son la incompatibilidad de muchas de sus piezas con el resto de las unidades que se pueden adquirir para uso privado, además existe una ausencia de registro por parte de los usuarios, el sistema más representativo y que actualmente continúa operando es "Bycyklen en Copenhague, Dinamarca, la cual es llamada la ciudad de las bicicletas (IDEA, 2012:78)", ya que más del 60\% de la población se desplaza por este medio.

3ํㅡㄹ Generación, incluida dentro de una proyección urbana en planes y programas para la búsqueda de una movilidad alternativa y sustentable, la cual cuenta con una tecnología más moderna, es necesario realizar un registro, se lleva un control de los beneficiarios y tiene una cuota por el uso de las bicicletas, es un sistema más operacional, se comenzó a implementar a partir del año 2007, esta generación es la más utilizada en las grandes ciudades a nivel mundial. Son proyectos costosos, de una gestión compleja y que presentan una rentabilidad económica a medida que la red supera cierta escala.

La tercera generación, Ecobici, se caracteriza por tener un "registro de usuarios, así como el pago de un depósito y el registro al utilizar Smart cards, micro chips o GPS, para el seguimiento y mantenimiento. Se colocan en cierto número de estaciones bajo las cuales la bicicleta está anclada a un panel inteligente que se libera cuando el usuario desliza su tarjeta, (ITDP, 2010:41)". Otro rasgo característico es que requiere de pocas personas para su operación y prestación del servicio, un ejemplo es Velib`en Paris, y Ecobici en la Ciudad de México, el sistema permite el alquiler público de bicicletas de manera más fácil para el usuario y con seguridad para el programa, convirtiendo a la bicicleta en un sistema público individual multimodal, ya que se puede complementar con el transporte público como el metro o el autobús.

4ํㅡㄹ Generación; técnicamente es el mismo sistema de la anterior generación, pero éste busca fomentar la intermodalidad con el resto de la oferta de transporte público, con esquemas tarifarios compartidos, es el sistema más novedoso, aun cuando no existe ninguna ciudad en donde se cuente 


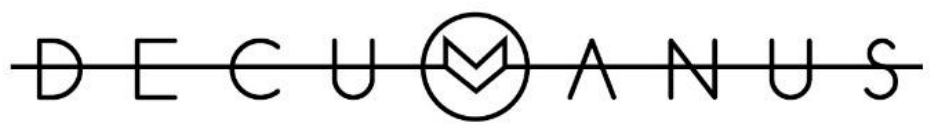

REVISTA INTERDISCIPLINARIA SOBRE ESTUDIOS URBANOS

con una cobertura al 100\%, inició operaciones en 2009, se caracteriza por ser un "sistema que incorpora la tecnología desde el registro hasta el seguimiento de las bicicletas por GPS y SMS, o bien por medio de una tarjeta con chip (ITDP, 2010:41). Fomenta la integración a otras modalidades de transporte público como la anterior generación, un ejemplo es "Call a Bike” en Alemania, el cual funciona mediante el envío de un SMS a través de un teléfono celular para desbloquearla. En la Ciudad de México ya se están implementando este tipo de tecnologías en la IV fase, así como en cicloestaciones de gran afluencia turística, se tiene el objetivo que todo el sistema Ecobici a largo plazo sea de $4^{a}$ generación.

\section{Características generales e importancia de los Sistema de Bicicletas Públicas}

La bicicleta se caracteriza por movilizar a individuos mediante su propia energía, es un medio de transporte limpio, rápido, económico. Por ello a nivel mundial los gobiernos están impulsando estrategias y líneas de acción para promover el uso de la bicicleta mediante los sistemas públicos, "en la actualidad en más de 150 ciudades en el mundo los han adoptado, (Gobierno de España, 2007:22) "un claro ejemplo es B`easy en Santiago de Chile, Samba en Río de Janeiro, Vélib en Paris la capital de Francia y Bicing en Barcelona, las cuales surgen por la necesidad de tener medios de transporte alternativos no motorizados y de carácter sustentable.

Para implementar un SBP se requiere de bicicletas, cicloestaciones, el elegir una ubicación adecuada de las bicicletas, los sistemas de rastreo y registro, el diseño, el mantenimiento y la redistribución de las bicicletas, como se puede apreciar en la tabla 1.

\begin{tabular}{|l|l|}
\hline Elemento & Características \\
\hline Bicicletas & Livianas, cómodas y adaptables \\
\hline Estaciones & $\begin{array}{l}\text { El modelo depende del esquema de } \\
\text { servicio que proporcione }\end{array}$ \\
\hline Ubicación de las bicicletas & $\begin{array}{l}\text { Establecidas en lugares estratégicos de } \\
\text { la ciudad }\end{array}$ \\
\hline Sistemas de rastreo & Por GPS \\
\hline Sistemas de registro & $\begin{array}{l}\text { Por medio de una cuenta de celular o } \\
\text { tarjeta de crédito }\end{array}$ \\
\hline Diseño & Especifico y exclusivo \\
\hline Mantenimiento & Siempre debe incluir mantenimiento \\
\hline
\end{tabular}




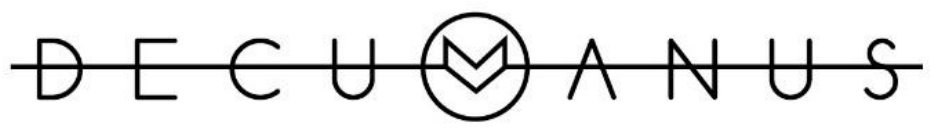

\begin{tabular}{|l|l|}
\hline Redistribución de las bicicletas & $\begin{array}{l}\text { Estrategia para buscar un balance de } \\
\text { flota }\end{array}$
\end{tabular}

Tabla 1. Aspectos más relevantes de un sistema SBP. Fuente: Elaborado con base a, "Estructura base para la implementación de sistemas de bicicletas públicas en España" (Acero, Jesús. Clean Air Institute, España, 2012:13)

En este sentido, Barragán (2010:55) define a los sistemas de bicicletas públicas como los "servicios de préstamos de bicicletas dentro de los núcleos urbanos, promovidos por los gobiernos. Están diseñados para suministrar servicios de movilidad eficiente y rápida de uso habitual y es considerado como un transporte público individual". Son "sistemas de contratación o préstamo gratuito de bicicletas dentro de zonas urbanas, implementados generalmente por los gobiernos. Se diferencian de los servicios habituales de préstamo de bicicletas, más enfocados al ocio o al turismo, por el hecho de prestar un servicio de desplazamientos eficiente, rápido y pensado para el uso cotidiano, (Gobierno de España, 2007:22)".

Por ello los SBP se pueden utilizar tanto en trayectos monomodales entre dos puntos o como extensión de un viaje intermodal al combinarse con el transporte público, los sistemas de bicicletas públicas se implementan con el objeto de realizar una gran diversidad de actividades acorde a las diferentes necesidades de la población en las ciudades como la diversión, el ejercicio, la salud, o por buscar una alternativa de movilidad en distancias cortas por mencionar algunos. En la tabla 2, se resaltan algunos de los beneficios más sobresalientes tanto directos como indirectos en torno a la utilización de la bicicleta.

\begin{tabular}{|c|c|}
\hline B. Directos & B. Indirectos \\
\hline Incremento del uso de la bicicleta & Hacer el ciclismo más visible \\
\hline Opción de movilidad adicional & $\begin{array}{l}\text { Estimular el desarrollo de las } \\
\text { infraestructuras para la } \\
\text { bicicleta }\end{array}$ \\
\hline \multicolumn{2}{|l|}{ Prevención de la congestión } \\
\hline $\begin{array}{l}\text { Aumento del atractivo para los } \\
\text { turistas }\end{array}$ & $\begin{array}{l}\text { Ahorro en la construcción de } \\
\text { infraestructura para coches }\end{array}$ \\
\hline Oportunidades de publicidad & Imagen de la ciudad positiva \\
\hline Oportunidades de empleo & Reduce las emisiones de CO2 \\
\hline
\end{tabular}

Tabla 2. Beneficios directos e indirectos de los SBP. Fuente: ITDP (2010:41) Experiencias y lecciones de sistemas de transporte público en bicicleta, para América Latina. 


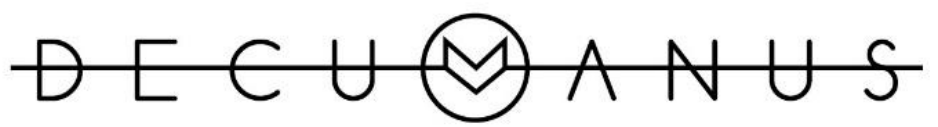

REVISTA INTERDISCIPLINARIA SOBRE ESTUDIOS URBANOS

Es importante señalar que los "SBP derivan de la implementación de planes y programas de movilidad urbana sustentable, en donde el principal objetivo radica en poner al servicio de los ciudadanos; bicicletas, estacionamientos e infraestructura vial en determinados espacios dentro de la ciudad, con el objeto de que la población acceda a bicicletas para llevar a cabo desplazamientos menores a 8 kilómetros y devolviéndolas en cicloestaciones" (Treviño, 2010:22).

Los SBP son automáticos y no requieren de un amplio equipo para su operación, solo el requerido para su mantenimiento y distribución, así como de personal para su admisión al sistema. La necesidad de movilidad es una de las necesidades básicas de las modernas sociedades que tienen ya conciencia del riesgo que implica un desarrollo urbano no controlado, el uso del automóvil como uno de los elementos más contaminantes, las necesidades de un traslado más eficaz y rápido y el derecho a gozar de una ciudad, es por ello que "los SBP proporcionan una nueva oferta de movilidad urbana flexible, cómoda, sana, rápida, y con un sentido intermodal al combinarse con el transporte público" (Treviño, 2010:15).

Así, hoy por hoy, el uso de la bicicleta ha dejado de ser una moda para convertirse en uno de los principales medios de transporte en las zonas metropolitanas, como la Ciudad de México, en la cual diariamente se realizan más de 100,000 viajes en bicicleta ${ }^{1}$, ciertamente la bicicleta tiene que ser considerada como un vehículo más en la calle con derechos y obligaciones.

\section{Ventajas de los SBP como medio de Movilidad No Motorizada (MNM)}

Los SBP "traen consigo ventajas dentro de la MNM, ya que, en las ciudades con un proceso de urbanización no controlado, el impulso a la movilidad no motorizada (MNM) en bicicleta, para ello es pertinente señalar al programa Ecobici, el cual constituye un medio de transporte económico, eficaz y sustentable" (Treviño, 2010:27), esto se explica por los beneficios económicos, sociales y ambientales, más aún cuando se utiliza de forma cotidiana, el uso más óptimo es buscar un equilibrio entre combinar el caminar (para trayectos cortos), el uso de la bicicleta (para trayectos intermedios) y utilizar el transporte público (para trayectos largos), con ello se lograría que "el transporte urbano se transformara en un medio de transporte más sostenible en términos económicos, sociales y ambientales" (IDEA 2007:33), pero además "fomenta la intermodalidad, al hacerla rápida y flexible, agiliza los tiempos de desplazamientos y reduce la necesidad de espacio en las ciudades, tanto por motivos de circulación como de estacionamiento; los usuarios no se preocupan por el mantenimiento de las bicicletas públicas en comparación con el uso de una bicicleta privada" (Treviño, 2010:24).

${ }^{1}$ https://www.ecobici.df.gob.mx/ 


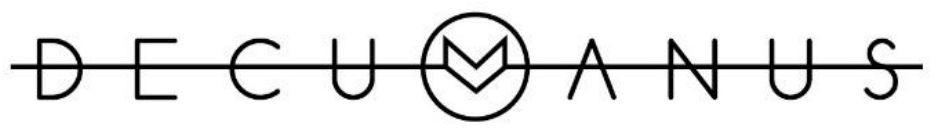

REVISTA INTERDISCIPLINARIA SOBRE ESTUDIOS URBANOS

Sin embargo, es necesario tener en claro que a corto plazo las bicicletas no resolverán por si solas los problemas de movilidad, "pero el hecho de desplazarse en bicicleta puede ser una de las maneras en que las personas pueden incidir directamente en mejorar su calidad de vida, alcanzar un progreso en su movilidad y propugnar por ciudades más sostenibles" (Treviño, 2010:12). Es importante señalar que los SBP tiene ventajas dentro de tres rubros: en el ambiental, en la salud y en la economía.

\section{Desventajas de los SBP como medio de Movilidad No Motorizada (MNM)}

En contraparte es importante identificar las desventajas y debilidades de los SBP, si bien a nivel mundial una gran parte de los SBP efectuados en la última década tienen la característica de ser concesionados a empresas privadas de carácter multinacional dedicadas a la publicidad, mismas que "han entrado en una férrea competencia por la provisión de servicios de bicicletas públicas" (Perez, Ruth, 2011:26) vinculados, en la mayoría de los casos, a contratos de explotación de espacio de publicidad. Dentro de estas empresas cabe señalar a Clear Channel de Estados Unidos, Cemusa de España o JCDcaux de Francia.

Estas concesiones en la mayoría de los países en donde se han implementado son "poco transparentes al hacerse con el servicio, esto a razón de que se cubren costos y beneficios" (Bea, 2009:29). Otra desventaja más a señalar es que en la mayoría de los casos son acuerdos limitados a un máximo de 10 años de operación, con lo cual "no garantiza su operatividad en el largo plazo, sumado a que en ningún caso se ven obligadas a pagar algún costo por el uso y utilización del suelo urbano" (Bea, 2009:12), es por esa razón que estas compañías suelen estar interesadas en grandes urbes en las cuales puedan obtener altos ingresos.

Otro inconveniente más es que "no existe la libre competencia, ya que cada empresa cuenta con un sistema de bicicletas y cicloestaciones compatible solo con su modelo, por ello las futuras ampliaciones estarán sujetas a ser llevadas a cabo por la misma empresa. El bajo costo por este tipo de servicio esconde un gran negocio muy rentable para las grandes multinacionales" (Bea, 2009:28), ello puede generar la pérdida de una oportunidad para la promoción de un transporte público sustentable.

Por otra parte, es importante señalar "la ausencia de una cultura vial y de respeto que se manifiesta, dentro del espacio urbano" (Treviño, 2010:19), en la indiferencia por parte de la población y de los automovilistas, en la poca eficacia en el trazado de las rutas, y la falta de seguridad en los 


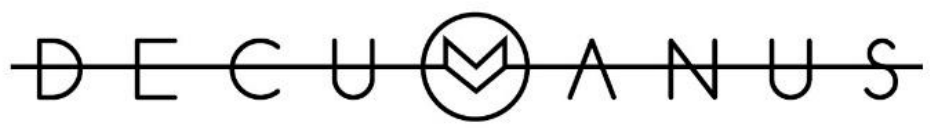

REVISTA INTERDISCIPLINARIA SOBRE ESTUDIOS URBANOS

corredores y ciclovías. Pese a estas condiciones los SBP como parte de la MNM está cobrando un gran impulso a escala global.

\section{Los SBP un nuevo paradigma urbano}

A manera de conclusión es de destacar la necesidad de un cambio de paradigma en torno a la movilidad, los SBP han cambiado el panorama, ya que es mediante la bicicleta que se recupera tiempo en el traslado, se tiene un ahorro económico y disminuye el stress de poseer un auto y contribuye a mejorar la salud. Al construir infraestructura ciclista es importante no solo la planificación e implementación aislada, como una ciclovía o un corredor, sino que es necesaria la planificación de una red ciclista a gran escala en la ciudad, por ello es apremiante considerar a la movilidad como un derecho que el Estado deberá garantizar, e impulsar el uso de un transporte público masivo y el transporte no motorizado.

Ciertamente las bicicletas públicas están en auge a nivel mundial, se están convirtiendo en una alternativa real de transporte urbano en distancias cortas en la zona central de las ciudades, por ello la implementación de los SBP no son el remedio a los problemas de movilidad urbana y regional, para que éstos sean eficaces tienen que estar integrados a una estrategia global de transporte. Por poner un ejemplo "en los últimos años la bicicleta ha ganado terreno en la Ciudad de México"2, teniendo impactos favorables en la recuperación del espacio público. Aquí surge la pregunta ¿faltan políticas públicas que promuevan realmente el transporte no motorizado como una alternativa real de movilidad en la Ciudad de México?, ¿las políticas implementadas en torno a la Movilidad No Motorizada en la Ciudad de México son aisladas y poco efectivas? Si bien se están sentando las bases para la promoción de la bicicleta como un medio alternativo de transporte, aún falta mucho por hacer, ya que algunos de los programas que se han implementado son de forma aislada como el cierre de la circulación en algunas avenidas los domingos, no son acciones que realmente incidan de forma importante en la movilidad en la Ciudad de México.

Por ello es apremiante la implementación de políticas públicas y programas que busquen la equidad en el transporte y la movilidad, cabe subrayar que la puesta en marcha de políticas y programas encaminados a impulsar la Estrategia de Movilidad no Motorizada en bicicleta en la Ciudad de México son consideradas acciones que no modifican radicalmente los patrones de movilidad actuales. Esto se explica ya que tanto el gobierno central como el delegacional tienen mesura al

\footnotetext{
2 SEDEMA llevo a cabo dicho conteo mediante ISA, S.C. "el Conteo de línea base de ciclistas en el Distrito Federal para la Secretaría de Medio Ambiente
} del Gobierno del Distrito Federal (diciembre de 2008)". 


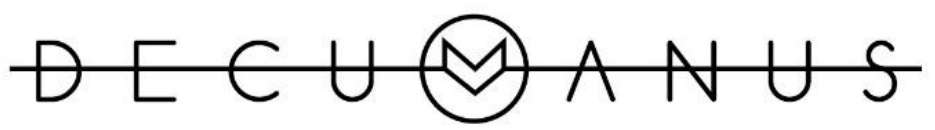

REVISTA INTERDISCIPLINARIA SOBRE ESTUDIOS URBANOS

implementar estas medidas a razón de poder perder electores con la ejecución de medidas más radicales, aunado a que, para dichos ámbitos gubernamentales, los ciclistas no son prioridad, ni el impulsar la bicicleta como un medio eficaz de transporte en distancias cortas. Resultado de ello, los administradores públicos prefieren promover acciones poco controvertidas, como los paseos ciclistas y los cierres dominicales, las cuales no cambian estructuralmente la forma de moverse. Es necesario cambiar la visión de muchas autoridades que creen falsamente que el fomento a los SBP, y que la bicicleta solamente cuenta con un carácter deportivo o recreativo; o mediante acciones como el cierre de algunas vialidades los domingos, o intervenciones desarticuladas entre sí. Por ende, es necesario que realmente se impulsen los SBP de forma integral y mediante la asignación suficiente del presupuesto.

\section{Bibliografía}

Acero, J. (2012). "Estructura base para la implementación de sistemas de bicicletas públicas en España". Clean Air Institute, 13.

Bea, M. (2009). "Los sistemas de bicicletas públicas urbanas". Universidad Autónoma de Barcelona, pp.17-20.

Barragán, F. (2010). "Sistemas de Bicicletas públicas en Europa". Seminario de Economía del transporte. Universidad Politécnica de Madrid, 55.

Ballén D.F. (2007, diciembre). Derecho a la Movilidad, en Derechos y Valores. Red de Revistas Científicas de América Latina, el Caribe, España y Portugal, num. 20, 169.

Borja, J. (2001). "Decálogo para un desarrollo urbano afortunado en Kit de Ciudades y medio ambiente". Guía práctica para la protección ambiental de los municipios de América Latina, Federación Mundial de Ciudades Unidas, 81.

Borja .J \& M. (2007). "El espacio púbico, ciudad y ciudadanía”. Barcelona, 30. 


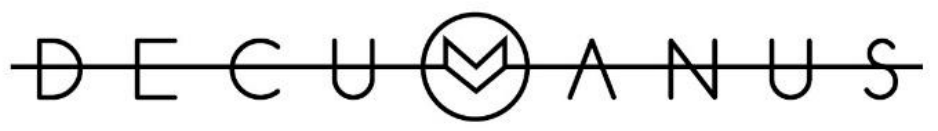

REVISTA INTERDISCIPLINARIA SOBRE ESTUDIOS URBANOS

Connolly, P. (2010). "La pérdida de Movilidad irregular y el orden urbano en la ZMVM 1990-2005 en: Ciudad de México: La gestión incluyente en las grandes ciudades”. Estructura urbana, Movilidad, seguridad y pluriculturalidad.

Esteban, A. (1994). “El eterno debate de la movilidad y la accesibilidad”, Madrid, 39.

Instituto para la Diversificación y Ahorro de Energía. (2007). "Guía metodológica para la implementación de sistemas de bicicletas públicas en España”. Instituto para la Diversificación y Ahorro de Energía. Madrid, 22.

Hoffmann; Odile \& Salmerón. (1997). “Entre representación y apropiación, las formas de ver y hablar el espacio". Nueve estudios sobre el espacio. Representación y formas de apropiación, CIESAS, México, 17.

IDEA. (2012). "Guía metodológica para la implantación de sistemas de bicicletas públicas en España”, 78.

ITDP. (2010). "Experiencias y lecciones de sistemas de transporte público en bicicleta para américa latina", 41.

Massey; Doreen, B. (2007). “For space”. Sage Publications. California, 79.

Moctezuma, R. (2003). "Ciudad y transporte: una perspectiva en Ciudad inclusiva". CEPAL, Chile, 175189.

Montañez; Gustavo \& Delgado. (1997). “Omar, Espacio, Territorio y Región, Conceptos básicos para un proyecto nacional". Revista Cuadernos de Geografía, Vol. 7 Número 2. Departamento de Geografía, Universidad Nacional de Colombia, Bogotá, 122.

Parrado, C. (2010). “Movilidad Sostenible”, Congreso sobre Ecología y ciudad, Barcelona, España, 22.

Pérez, López R. (2011). "Por mi ciudad en bicicleta, experiencias ciclistas en la ciudad de México", Bicitekas A.C., México.

Unikel L., Ruiz, C. \& Garza, G. (1978). “El desarrollo urbano de México”, COLMEX, México, 119. 